\title{
BMJ Global Health Making sense of emerging evidence on the non-specific effects of the BCG vaccine on malaria risk and neonatal mortality
}

\author{
Quique Bassat (1D , ${ }^{1,2,3,4,5}$ Gemma Moncunill, ${ }^{1}$ Carlota Dobaño ${ }^{1}$
}

\begin{abstract}
To cite: Bassat Q, Moncunill G, Dobaño C. Making sense of emerging evidence on the non-specific effects of the $B C G$ vaccine on malaria risk and neonatal mortality. BMJ Global Health 2020;5:e002301. doi:10.1136/ bmjgh-2020-002301
\end{abstract}

Received 8 January 2020 Revised 8 February 2020 Accepted 10 February 2020

\section{SLinked}

- http://dx.doi.org/10.1136/ bmjgh-2019-001873

- http://dx.doi.org/10.1136/ bmjgh-2019-001862

Check for updates

(C) Author(s) (or their employer(s)) 2020. Re-use permitted under CC BY-NC. No commercial re-use. See rights and permissions. Published by BMJ

For numbered affiliations see end of article.

Correspondence to Dr Quique Bassat; quique.bassat@isglobal.org
Vaccines are, indisputably, one of the greatest public health interventions, with a substantial positive impact on child survival. The remarkable declines in child mortality observed during the last quarter of a century, whereby global under 5 deaths were essentially halved, go hand in hand with the estimated 2-3 million child deaths prevented by vaccines annually. ${ }^{1}$ The premise for this is clear: vaccines directly prevent a variety of life-threatening diseases. Vaccines can also be held directly responsible for the eradication of smallpox, the first and only infectious disease extinguished by the action of humans and are paving the way for the disappearance of other terrible infections such as polio, measles or rubella.

In recent years, however, it has become increasingly clear that the impact of vaccines is achieved by their direct prevention against specific pathogens, and through a series of non-specific effects. ${ }^{2}$ These non-specific effects, also termed 'heterologous' effects, appear to be more common as a result of the vaccination with certain live-attenuated antigens (eg, the Bacillus Calmette-Guérin (BCG), measles or polio), and have been proposed for a wide variety of existing vaccines. Observational studies have pointed out to a longerterm all-cause mortality decrease attributable to having received those vaccines, independent of the target disease. Non-specific effects are understandably less tangible and less wellcharacterised than the direct ones, and therefore, remain a matter of significant debate and controversy. ${ }^{3}$

To date, the nature of non-specific effects has not been fully elucidated, although the current thinking points to trained innate immunity as the main underlying mechanism. ${ }^{4}$ Trained immunity refers to an immunological memory of the innate response, a process in which certain stimuli induce epigenetic changes in the innate immune cells, ${ }^{5}$ increasing the response to the same and different subsequent stimuli. Vaccines with non-specific effects would induce reprogramming of the innate immune responses, a mechanism that clearly differs from the adaptive immunity induced by the antigenspecific responses to the vaccine. The uncertainty regarding their real added benefit calls for observational studies and clinical trials to help shed some more light on their true nature, and biological and immunological mechanisms.

Two studies published recently in $B M J$ Global Health ${ }^{6} 7$ are now providing further evidence of the non-specific effects of one live attenuated vaccine, namely BCG, ideally administered immediately after birth to protect against tuberculosis. Both articles appear to underscore malaria (and its deleterious direct effect during infancy, or indirect one when acquired during the mother's pregnancy) at the core of the preventative immunomodulatory non-specific effects conferred by the BCG vaccine.

Jensen $e a^{6}$ dig into the data derived from three randomised controlled trials conducted in Guinea-Bissau during 12 consecutive years and involving over 6500 infants with low birth weight (LBW). In those studies, the administration of early BCG (as opposed to after a few weeks) was associated with an overall $38 \%$ reduction in all-cause neonatal mortality and a $16 \%$ reduction in infant mortality. ${ }^{8}$ The aim was to evaluate whether seasonal patterns of the mortality prevented could shed some light into the epidemiological aspects of nonspecific effects, and the particular effect on diseases other than tuberculosis. Their analyses highlight a stronger benefit in relation to all-cause neonatal mortality when administration of BCG coincided with the malaria 
peak-season (November to January), with a much more modest effect during the rest of the year.

The authors hypothesise that, similar to what other studies have shown, such an effect in overall neonatal survival could be achieved through a non-specific protection against malaria. This was supported by additional laboratory work conducted among a subgroup $(n=467)$ of vaccinated newborns who evidenced stronger proinflammatory responses to heterologous challenge. Their hypothesis would be explained by the immunomodulatory non-specific effects of the BCG vaccine against the life-threatening consequences of placental malaria, which is known to cause an enhanced risk of subsequent morbidity and mortality in the neonatal period. ${ }^{9}$ Thus, BCG vaccination would confer an increased resilience against the risk of infection during the first months of life by ameliorating the detrimental immunological effects of malaria in the later stages of pregnancy.

A few considerations appear important when analysing such hypothesis. On the one hand, the population chosen for this analysis (LBW babies $<2500 \mathrm{~g}$, enrolled in the clinical trials) appears most adequate, because of a variety of reasons. Indeed, in Guinea-Bissau, LBW newborns were deliberately left out of BCG early vaccination, even though they were probably those who could benefit the most from more proactive interventions, considering that LBW confers a significant risk for neonatal and infant death, with malaria during pregnancy being considered a key risk factor. ${ }^{10}$

On the other hand, the coincidence of the administration of the vaccine with malaria season necessarily implies that the effects occurring during pregnancy in terms of creating an adverse immune phenotype in the fetus (that the non-specific effects of the vaccine would somehow 'correct') may have predominantly affected mothers during their latest stages of pregnancy, so as to also occur during the high malaria season. The confirmation that this was the case, and that newborns of primigravidae appeared significantly more protected by BCG than those of multigravidae (also a phenotype typical of malaria during pregnancy) is reassuring and further supportive of the link with malaria.

Finally, when looking at the likely causes of death as determined by verbal autopsy and clinical records it seems majorly attributed to septicaemia and respiratory infections, in spite of the inherent weaknesses of the methods used for establishing cause of death. Therefore, it would appear that the increased risks conferred by malaria in pregnancy would not necessarily be predominantly due to malaria (congenital or acquired) at an early stage of life (a rarer event in malaria-endemic areas, in comparison to malaria at later stages during infancy ${ }^{11}$ but rather to a more generic 'infectious' risk during those very first weeks. Studies have demonstrated a higher incidence of infectious episodes (particularly of respiratory and gastrointestinal nature) in areas with high prevalence of placental malaria, ${ }^{12}$ and it is also well known that malaria can be associated with an increased risk of secondary bacterial infections. ${ }^{13}$
Other live-attenuated vaccines, such as measles, have also been associated with non-specific effects in the risk of sepsis and acute lower respiratory tract infections. ${ }^{14}$ Irrespective of the true mechanisms underscoring such associations, the work presented establishes a plausible and interesting hypothesis which will need future testing (ideally in other malaria endemic areas) and merits further consideration.

In the second article, Berendsen et al present very convincing and complementary observational data on the alleged protection of BCG vaccination against malaria infection and disease among children under five in sub-Saharan Africa. Although many studies had analysed the potential non-specific effects of vaccines against other infections, scarce and contradictory data were available regarding the potential non-specific benefits in terms of malaria risk at a population level. Their analysis, more straightforward than that presented in the first article, used Demographic Health Survey data from 13 sub-Saharan countries involving over 34200 children.

Berendsen $e t a l^{7}$ found that among children with confirmed BCG vaccination (as documented in the child's health card), the risk of malaria was significantly lower (AOR $0.88,95 \%$ CI 0.82 to 0.94 ). Such an association was stronger in areas of suboptimal BCG coverage, indicated a similar prevention of both symptomatic and asymptomatic infections, and appeared consistent irrespective of the age at which the infant was vaccinated (with perhaps an increasing benefit with increasing infant's age).

The authors contextualise their findings in the current scenario whereby the RTS,S/AS01E malaria vaccine, the first vaccine found to have a direct effect on the risk of human malaria, is being cautiously implemented in a pilot phase in three African countries under strict scrutiny both because of its suboptimal efficacy and potential safety concerns observed in the phase 3 trial. Interestingly, such safety signals (essentially an increase in the risk of meningitis and cerebral malaria among a specific group of the vaccinated older children with the RTS,S, as compared with their respective controls) have been proposed to derive from the non-specific effects of the control vaccine (live attenuated rabies vaccine) rather than resulting from the deleterious effect of RTS,S. ${ }^{15}$ The authors conclude that the non-specific effects of the BCG vaccine against malaria, particularly in areas of low vaccination coverage, could potentially reduce up to $9.6 \%$ the malaria prevalence, and that such impact could be synergistic with the other malaria control tools, including the use of the RTS,S vaccine, and would be highly costeffective in the global fight against malaria.

The evidence supporting the somehow ambiguous non-specific effects of vaccines is slowly building up. The robust data provided by the aforementioned two articles on the non-specific effects associated to BCG opens new avenues for future research and corroboration, ${ }^{16}$ and further strengthen our current understanding that vaccines, beyond preventing those diseases they are intended to prevent, can indirectly produce many 
additional public health benefits. This opens the door to the design of vaccines targeting a broad spectrum of diseases. In this dangerous era of vaccine hesitancy, such evidence needs to be treasured and widely disseminated.

\section{Author affiliations}

${ }^{1}$ ISGlobal, Hospital Clínic - Universitat de Barcelona, Barcelona, Spain

${ }^{2}$ Centro de Investigação em Saúde de Manhiça (CISM), Manhiça, Maputo, Mozambique

${ }^{3}$ ICREA, Pg. Lluís Companys 23, Barcelona, Spain

${ }^{4}$ Pediatric Infectious Diseases Unit, Pediatrics Department, Hospital Sant Joan de Déu (University of Barcelona), Barcelona, Spain

${ }^{5}$ Consorcio de Investigación Biomédica en Red de Epidemiología y Salud Pública (CIBERESP), Madrid, Spain

Contributors QB wriote the first draft of this editorial whioch was significantly reviewed by $\mathrm{CD}$ and $\mathrm{GM}$.

Funding The authors have not declared a specific grant for this research from any funding agency in the public, commercial or not-for-profit sectors.

Competing interests None declared.

Patient consent for publication Not required.

Provenance and peer review Commissioned; internally peer reviewed.

Data availability statement № additional data are available.

Open access This is an open access article distributed in accordance with the Creative Commons Attribution Non Commercial (CC BY-NC 4.0) license, which permits others to distribute, remix, adapt, build upon this work non-commercially, and license their derivative works on different terms, provided the original work is properly cited, appropriate credit is given, any changes made indicated, and the use is non-commercial. See: http://creativecommons.org/licenses/by-nc/4.0/.

ORCID iD

Quique Bassat http://orcid.org/0000-0003-0875-7596

\section{REFERENCES}

1 World Health Organization. Immunization factsheet. Available: https://www.who.int/news-room/facts-in-pictures/detail/ immunization [Accessed 19 Dec 2019].
2 de Bree LCJ, Koeken VACM, Joosten LAB, et al. Non-Specific effects of vaccines: current evidence and potential implications. Semin Immunol 2018;39:35-43.

3 Pollard AJ, Finn A, Curtis N. Non-Specific effects of vaccines: plausible and potentially important, but implications uncertain. Arch Dis Child 2017;102:1077-81.

4 Blok BA, Arts RJW, van Crevel R, et al. Trained innate immunity as underlying mechanism for the long-term, nonspecific effects of vaccines. J Leukoc Biol 2015;98:347-56.

5 Netea MG, Joosten LAB, Latz E, et al. Trained immunity: a program of innate immune memory in health and disease. Science 2016;352:aaf1098.

6 Jensen K, Biering-Sørensen S, Ursing J, et al. Seasonal variation in the non-specific effects of BCG vaccination on neonatal mortality: three randomized controlled trials in Guinea-Bissau. BMJ Glob Health 2020:e001873.

7 Berendsen ML, van Gijzel SW, Smits J, et al. BCG vaccination is associated with reduced malaria prevalence in children under the age of 5 years in sub-Saharan Africa. BMJ Glob Health 2019;4:e001862.

8 Biering-Sørensen S, Aaby P, Lund N, et al. Early BCG-Denmark and Neonatal Mortality Among Infants Weighing <2500 g: A Randomized Controlled Trial. Clin Infect Dis 2017;65:1183-90.

9 Bardají A, Sigauque B, Sanz S, et al. Impact of malaria at the end of pregnancy on infant mortality and morbidity. $J$ Infect Dis 2011;203:691-9.

10 Eisele TP, Larsen DA, Anglewicz PA, et al. Malaria prevention in pregnancy, birthweight, and neonatal mortality: a meta-analysis of 32 national cross-sectional datasets in Africa. Lancet Infect Dis 2012;12:942-9.

11 Ceesay SJ, Koivogui L, Nahum A, et al. Malaria prevalence among young infants in different transmission settings, Africa. Emerg Infect Dis 2015;21:1114-21.

12 Rachas A, Le Port A, Cottrell G, et al. Placental malaria is associated with increased risk of nonmalaria infection during the first 18 months of life in a Beninese population. Clin Infect Dis 2012;55:672-8.

13 Church J, Maitland K. Invasive bacterial co-infection in African children with Plasmodium falciparum malaria: a systematic review. BMC Med 2014;12:31.

14 Aaby P, Martins CL, Garly M-L, et al. Non-Specific effects of standard measles vaccine at 4.5 and 9 months of age on childhood mortality: randomised controlled trial. BMJ 2010;341:c6495.

15 Gessner BD, Knobel DL, Conan A, et al. Could the RTS,S/AS01 meningitis safety signal really be a protective effect of rabies vaccine? Vaccine 2017;35:716-21.

16 Covián C, Fernández-Fierro A, Retamal-Díaz A, et al. Bcg-Induced cross-protection and development of trained immunity: implication for vaccine design. Front Immunol 2019;10:2806. 